\section{MANAGEMENT OF PREGNANCY IN A 27 YEAR OLD MULTIGRAVIDA WOMAN WITH CERVICAL CANCER IN RESOURCE LIMITED SETTINGS: A CASE REPORT}

V Belivia Tripuspita*, S Patricia Mongan, J Joice Kaeng. Manado, Manado, Indonesia

\subsection{6/ijgc-2021-ESG0.30}

Introduction/Background* Cervical cancer is the most common gynecologic cancer in women worldwide, and is a global problem. Cervical cancer occurs mainly in developing countries and low-income countries. In Indonesia, cervical cancer has the highest percentage of cancer in 2013. The number of women with cervical cancer ranges from 90-100 cases per 100,000 population and every year there are over 40.000 cervical cancer cases. Its management is challenging especially in pregnancy. We report management of pregnancy in a 27 year old woman with cervical cancer in Manado, Indonesia.

Methodology none

Case report A 27-year-old Indonesian women gravida 6, para 2 , abortus 3 at 10-11 weeks of gestational age (GA) came to Prof. DR. R. D. Kandou General Hospital complaining of vaginal bleeding mixed with foul smell discharges for 4 months. Speculum examination revealed visible exophytic mass measuring $\pm 5 \times 4 \times 5 \mathrm{~cm}$ suspiciously originating from the portio extending to the $1 / 3$ proximal of the vagina that are friable and bleed easily. Histological diagnoses were moderately differentiated invasive squamous cell carcinoma of the cervix with obvious parametrial involvement (stage IIB). The patient received 4 series of neoadjuvant chemotherapy (NACT) with carboplatin and paclitaxel. The patient tolerated the therapy well and had a good response, no obvious side effect was observed. The Caesarean section $(\mathrm{C} / \mathrm{S})$ were done at 36 weeks GA, a live baby boy of 2000 grams weight was extracted with APGAR score 6-8.

Result(s)* none

Conclusion* At present, the consensus and guidelines for cervical cancer treatment in pregnancy are still uncertain, but this case suggest the combination of carboplatin and paclitaxel could be effective and well-tolerated regimen against stage IIB cervical cancer. Furthermore, this case illustrates the importance of screening for cervical cancer before pregnancy to prevent complications that may occur to both mother and child during pregnancy.

\section{PATTERN OF RELAPSE IN PATIENTS WITH STAGE IB1 CERVICAL CANCER AFTER RADICAL HYSTERECTOMY. MIS VS OPEN APPROACH. SYSTEMATIC REVIEW AND META- ANALYSIS}

${ }^{1} \mathrm{~N}$ Manzour*, ${ }^{1} \mathrm{~F}$ Boria, ${ }^{1} \mathrm{E}$ Chacon, ${ }^{2} \mathrm{~J}$ Nuñez, ${ }^{2} \mathrm{~N}$ Martín-Calvo, ${ }^{1} \mathrm{JA}$ Mínguez, ${ }^{1} \mathrm{D}$ Vazquez, ${ }^{1} \mathrm{~T}$ Castellano, ${ }^{1} \mathrm{~J}$ Vara, ${ }^{1} \mathrm{I}$ Brotons, ${ }^{3} \mathrm{JM}$ Aramendia, ${ }^{3} \mathrm{~A}$ Gonzalez-Martin, ${ }^{3} \mathrm{D}$ Salas, ${ }^{3}$ L Sánchez Lorenzo, ' $\mathrm{LM}$ Chiva, ${ }^{1} \mathrm{JL}$ Alcazar. ${ }^{1}$ Clinica Universidad de Navarra, Gynecology, Spain; ${ }^{2}$ Clinica Universidad de Navarra, Spain; ${ }^{3}$ Clinica Universidad de Navarra, Medical Oncology

\subsection{6/ijgc-2021-ESG0.31}

Introduction/Background* After the LACC trial, SUCCOR study and other studies, we know that patients who have undergone Minimally Invasive Surgery for cervical cancer have worse outcomes, but today we really don't know if the surgical approach can be a reason to change the pattern of relapses on these patients. We would try to evaluate the pattern of relapse in patients with cervical cancer Ib1 FIGO 2009 who underwent radical hysterectomy with different surgical approaches.

Methodology Systematic review of literature was performed in PubMed, Cochrane Library, Clinicaltrials.gov and Web of science with terms like 'pattern relapse cervical cancer,' 'pattern recurrence cervical cancer,' 'Open vs. Laparoscopic cervical cancer relapse.' Inclusion criteria were prospective or retrospective comparative studies to different surgical approaches that described patterns or locations of relapse in patients with Ib1 cervical cancer. For the quantitative analysis, the pool odds ratio of the recurrence localization was calculated. The quality of the studies was assessed with the Newcastle-Ottawa scale.
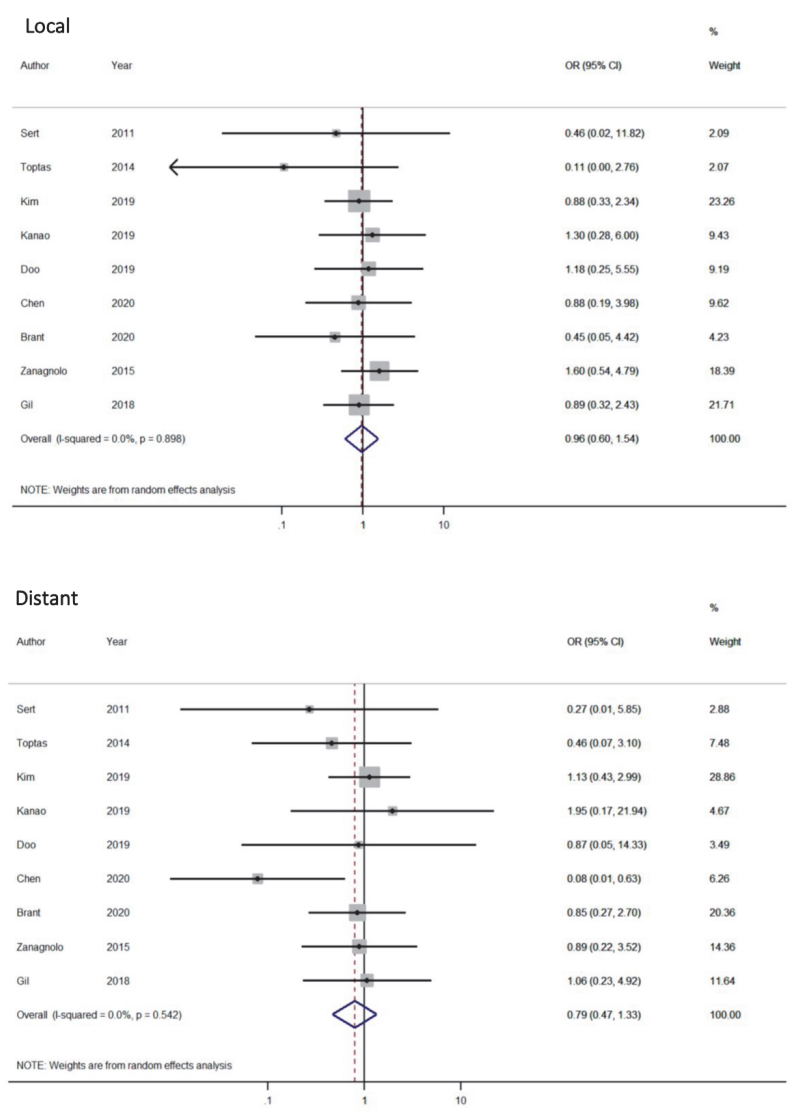

Abstract 445 Figure 1

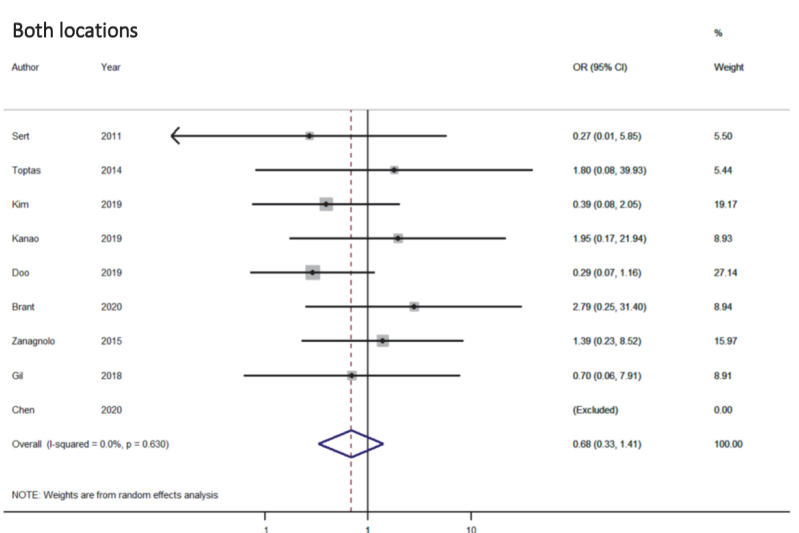

Abstract 445 Figure 2 
Result(s)* The research resulted in 782 eligible citations from January 2010 to October 2020. After the exclusion, nine articles that met all the inclusion criteria were included, comprising data from 1663 patients who underwent Radical Hysterectomy for Cervical Cancer IB1, the incidence of relapse was $10.6 \%$. Total patients who underwent open radical hysterectomy were $809(48.6 \%)$ with 75 relapses, patients who underwent minimally invasive surgery were 854 (51.4\%) and 105 relapses. When we compered the pattern of relapse local, Distant and Both of each group (open surgery and minimally invasive surgery), we did not see statistically significant differences (OD 0.963; 95\% CI, 0.602- 1.541; p=0.898), (OD $0.788 ; 95 \%$ CI, 0.467- 1.330; $\mathrm{p}=0.542$ ) and (CI 0.683; 95\% CI, 0.331- 1.407; $\mathrm{p}=0.630$ ) respectively.

Conclusion* There are no differences in patterns of relapse across surgical approaches in patients with stage IB1 cervical cancer undergoing radical hysterectomy as primary treatment.

\section{STAGE MIGRATION IN PATIENTS WITH LYMPH NODE POSITIVE CERVICAL CANCER}

${ }^{1} \mathrm{~T}$ Fahmy*, ${ }^{2} \mathrm{M}$ Persic. ${ }^{1}$ Barts and The London School of Medicine and Dentistry, United Kingdom; ${ }^{2}$ Royal Derby Hospital, United Kingdom

\subsection{6/ijgc-2021-ESGO.32}

Introduction/Background* Cervical cancer is the most common gynaecological malignancy worldwide. Despite strides in disease prevention with HPV (human papillomavirus) vaccination, and early detection of pre-cancerous changes, cervical cancer is nonetheless associated with high mortality. Survival is strongly linked to initial FIGO (International Federation of Gynaecology and Obstetrics) disease stage at diagnosis. In 2018, the FIGO staging criteria for cervical cancer were revised to include lymph node status, with positive nodes upstaging patients to stage $3 \mathrm{C}$ disease. This resulted in a retrospective stage migration for many patients. This study aims to analyse the effect of stage migration in cervical cancer on disease survival and systemic recurrence.

Methodology Data from a cohort of 76 cervical cancer patients from the University Hospital of Derby and Burton NHS Trust diagnosed and treated with chemoradiation and brachytherapy from 2012-2017 were collected. Patients with positive lymph nodes at diagnosis were assigned a new stage based on the current 2018 FIGO criteria and subsequently compared to patients whose stage at initial diagnosis remained unchanged.

Result(s)* $46 \%$ of patients were assigned a new higher stage based on lymph node status at diagnosis as per 2018 FIGO staging. An approximate $2.5 \mathrm{x}$ increase in cancer-related mortality was seen amongst those who had stage migrated versus those who remained the same stage $(37.14 \%$ versus $14.63 \%$, $p=0.024)$. Furthermore, a non-significant difference was seen in rates of systemic recurrence between the two groups, with around twice as many of the lymph node positive patients recurring within the timeframe of the study $(40.00 \%$ compared with $19.51 \%, p=0.05$ ).

Conclusion* These results reinforce the importance in the inclusion of lymph node status within 2018 FIGO staging criteria owing to the significant effect upon mortality in those who had stage migrated. The poorer prognosis and survival in the stage migration group also highlights the need for aggressive intervention in those with positive lymph nodes.

\section{DIAGNOSTIC PERFORMANCE OF PET-TC IN LYMPHATIC STAGING VS SURGICAL STAGING IN PATIENTS WITH LOCALLY ADVANCED CERVIX CANCER}

AF Rave Ramirez*, D González García-Cano, O Arencibia Sanchez, M Laseca Modrego, A Martín Martínez, T Benitez Delgado. Complejo Hospitalario Universitario Insular Materno Infantil de Gran Canaria, Gynecologic Oncology, las palmas de gran canaria, Spain

\subsection{6/ijgc-2021-ESG0.33}

Introduction/Background* The most important prognostic factor in cervical cancer is lymph node involvement. Up to $24 \%$ of patients with a negative CT scan show histological lymph node disease.The gold standard is histopathological study,but surgery is not exempt from possible complications (even laparoscopic approach). We intend to know the sensitivity and specificity of PET/CT in paraortic lymph node staging in LACC with negative CT.

Methodology Retrospective study of patients with LACC who have undergone laparoscopic paraortic lymphadenectomy and previously had performed a PET/CT. The indication for paraortic surgical staging in patients with LACC is a negative CT scan for disease at the paraortic level.

Result(s)* The mean age of the patients with cervical cancer was 52.7 years (range 23-97 years). 69\% were in advanced stages at the time of diagnosis. It should be noted that $35.5 \%$ of patients diagnosed in advanced stages underwent paraortic surgical staging. We have performed 227 laparoscopic paraortic lymphadenectomies in patients with LACC, 14\% with metastatic involvement. In 52 patients we had a PET/CT prior to paraortic lymphadenectomy. In the group of patients with negative PET ( $\mathrm{n}=45)$, we found that surgical staging was negative in $95.5 \%(\mathrm{n}=43)$. Meanwhile in patients with positive PET at the paraortic level $(n=7)$, we found that in 4 cases it was confirmed lymph node involvement in histology and in 3 cases they were false positives (predictive value $57.2 \%$ positive)

Conclusion* The specificity of PET/CT for paraortic lymph node staging in LCCA with Negative CT scan is $93.4 \%$, and the sensitivity is $66.6 \%$ in our series. If this trend is confirmed, we could consider modifying our strategy in the indication for paraortic surgical staging: in case of advanced stages with negative CT and PET/CT for lymphatic involvement, do not perform paraortic lymphadenectomy (negative predictive value of 95.5\%) and in cases of negative CT and positive PET, consider performing lymphadenectomy paraortic (positive predictive value of $57.2 \%$ ).

\section{ADJUVANT THERAPY AFTER RADICAL HYSTERECTOMY FOR EARLY CERVICAL CANCER. ARE WE HARMING OUR PATIENTS?}

F Boria*, N Manzour, E Chacon, N Martin, D Vazquez, T Castellano, JÁ Mínguez, JL Alcazar, A Gonzalez-Martin, L Sánchez Lorenzo, J Espinos, M Cambeiro, LM Chiva. Clinica universidad de navarra, Gynecologic Oncology, Spain

\subsection{6/ijgc-2021-ESG0.34}

Introduction/Background* In addition to positive pelvic lymph nodes, parametrial extension, and positive surgical margins, a combination of intermediate risk factors (Sedlis criteria) have been identified as poor prognostic factors. ${ }^{1-7}$ In these patients, adjuvant therapy is recommended in most of the clinical guidelines. However, indications for adjuvant therapy 